\title{
Performance comparison of zero-Schottky-barrier and doped contacts carbon nanotube transistors with strain applied
}

\author{
Md. Abdul Wahab ${ }^{1, *}$ and Khairul Alam²
}

Atomistic quantum simulation is performed to compare the performance of zero-Schottky-barrier and doped source-drain contacts carbon nanotube field effect transistors (CNTFETs) with strain applied. The doped source-drain contact CNTFETs outperform the Schottky contact devices with and without strain applied. The off-state current in both types of contact is similar with and without strain applied. This is because both types of contact offer very similar potential barrier in off-state. However, the on-state current in doped contact devices is much higher due to better modulation of on-state potential profile, and its variation with strain is sensitive to the device contact type. The on/off current ratio and the inverse subthreshold slope are better with doped source-drain contact, and their variations with strain are relatively less sensitive to the device contact type. The channel transconductance and device switching performance are much better with doped source-drain contact, and their variations with strain are sensitive to device contact type.

Keywords: Zero-Schottky-barrier; Doped contact; Strain; Inverse subthreshold slope; Intrinsic cut-off frequency

Citation: Md. Abdul Wahab and Khairul Alam, "Performance comparison of zero-Schottky-barrier and doped contacts carbon nanotube transistors with strain applied", Nano-Micro Lett. 2, 126-133 (2010). doi: 10.5101/nml.v2i2.p126-133

Carbon nanotube $(\mathrm{CNT})$ is a fascinating material that shows metallic or semiconducting behavior depending on its radius and chirality [1-7]. CNTs can withstand very large mechanical strains [8], and have extremely high Young's modulus [9]. The strain has significant effects on the electronic properties of CNTs [3-7,10-14]. Maiti et al. [14] have shown that strain can change the conductance of a zigzag nanotube by several orders of magnitude. The pioneer experiment by Tombler et al. [15] shows that high strain $(\sim 3 \%)$ can change the conductance of a metallic single-walled nanotube by two orders of magnitude. In that experiment, strain was applied to a suspended nanotube using atomic force microscope (AFM) tip. Strain can open up a band gap in metallic CNTs, and can modify the band gap of semiconducting CNTs [16]. Small band gap semiconducting or quasi-metallic nanotubes exhibit the largest changes in resistance and piezo-resistive gauge factors, and they can be used as nanoscale pressure sensors [17].

While the study of strain effects on electronic and mechanical properties of CNTs shows significant progress, strain engineering in CNT based devices is still in early stage. Single-walled carbon nanotube devices have been fabricated on elastomeric polydimethylsiloxane (PDMS) substrates [18]. In those devices, strain has been applied to modulate their electronic properties. The conductance of a suspended multi-walled CNT has been measured by applying strain using AFM tip [19]. The strain effects on the performance of ballistic Schottky-barrier carbon nanotube transistors have been theoretically studied [20].

In this paper, we compare the performance of ballistic zero-Schottky-barrier and doped source-drain contacts carbon 
nanotube transistors with strain applied. Performance comparison between Schottky and doped contacts CNTFETs has been carried out before without strain [21,22]. Our simulation model self-consistently solves the non-equilibrium Green's function (NEGF) equations for charge density and the two dimensional Poisson's equation in cylindrical coordinates for electrostatics. The CNT is modeled using $\pi$-orbital of carbon atom. The doped contact devices outperform the Schottky contact CNTFETs. The variation of off-state current with strain is not sensitive to the contact type; however, the magnitude of on-state current variation with strain is sensitive to device contact type. This is because the potential barrier in off-state for both types of contact is almost same, and therefore, the off-state current is not sensitive to the contact type with and without strain. However, the on-state current in Schottky contact devices is limited by the Schottky barrier, and the gate does not modulate much the potential profile when the potential under the gate goes below the source Fermi level. On the other hand, the gate modulation of potential profile is relatively larger in doped contact devices even after the source-channel flat band condition, and therefore, the on-state current in doped contact devices is much higher. The strain changes the band gap that changes the modulation of on-state band profile. This effect is higher in doped contact devices. The on/off current ratio and the inverse subthreshold slope are better in doped contact devices, and their variations with strain are not much sensitive to device contact type. The switching performance of doped contact devices is better, and its variation with strain is sensitive to device source-drain contact type.

\section{SIMULATION MODEL}

The simulation model uses a self-consistent solution between electrostatics and charge density. For a coaxially gated CNTFET, we obtain electrostatic potential by solving two-dimensional Poisson's equation in cylindrical coordinates $(r$, $\phi, z)$

$\frac{\partial^{2} V}{\partial r^{2}}+\left[\frac{1}{r}+\frac{1}{\varepsilon} \frac{\partial \varepsilon}{\partial r}\right] \frac{\partial V}{\partial r}+\frac{\partial^{2} V}{\partial z^{2}}=\frac{-\rho}{\varepsilon}$

The Poisson's equation, Eq. (1), is discretized using finite difference, and is solved by standard Newton-Raphson method. The potential is fixed to $V_{G S}-\Phi_{G} / q$ at the gate electrode. For Schottky contact, potential is fixed to $-\Phi_{S} / q$ at the source electrode and to $V_{D S}-\Phi_{D} / q$ at the drain electrode. Here, $V_{G S}$ and $V_{D S}$ are the gate to source and drain to source voltages, and $\Phi_{G}$,
$\Phi_{S}$, and $\Phi_{D}$ are the work functions of gate, source, and drain metallizations. For doped source-drain contacts, the axial component of electric field is set to zero at the source and drain ends. The radial component of electric field is set to zero along the exposed surface of dielectric.

The CNT is modeled using $\pi$-orbital basis of carbon atom. When strain is applied, we assume that the on-site energy does not change, and the hopping parameter changes following Harrison's formula [23] $V_{p p \pi}=V_{p p \pi}^{0}\left(r_{0} / r\right)^{2}$. Here, $V_{p p \pi}^{0}$ and $r_{0}$ are the hopping parameter and the carbon-carbon bond length, respectively, of the unstrained CNT, and $r$ is the bond length of strained CNT. With uniaxial strain applied, the axial, $r_{t}$, and the circumferential, $r_{c}$, components of a carbon-carbon bond are calculated by the following equations [5]

$$
\begin{aligned}
& r_{t}=\left(1+\varepsilon_{t}\right) r_{0 t} \\
& r_{c}=\left(1+\varepsilon_{c}\right) r_{0 c} .
\end{aligned}
$$

Here, axial strain, $\varepsilon_{t}$ and circumferential strain, $\varepsilon_{c}$ are related via Poisson's ratio $v=-\varepsilon_{c} / \varepsilon_{t}$, and $r_{0 t}$ and $r_{0 c}$ are the axial and circumferential components, respectively, of the unstrained carbon-carbon bond. A Poisson's ratio value of 0.2 is used in our simulation $[7,20]$. For torsional strain, the circumferential component of the carbon-carbon bond is modified as $r_{c}=r_{0 c}+$ $\tan (\gamma) r_{0 t}$ where, $\gamma$ is the shear strain. The Hamiltonian parameter values are taken from Ref. [24].

For charge density calculation, we use recursive Green's function (RGF) algorithm [25,26] to solve NEGF equations. The charge density at the $L^{\text {th }}$ atomic layer is calculated from

$$
\rho_{L}=(2 e) \int \frac{d E}{2 \pi} \operatorname{tr}\left\{f_{S} A_{L, L}^{L}+f_{D}\left[A_{L, L}-A_{L, L}^{L}\right]\right\},
$$

Where, the factor 2 includes spin degeneracy, $e$ is the electronic charge, $\operatorname{tr}$ is the trace over the atomic orbitals, and $f_{S}$ and $f_{D}$ are the source and drain Fermi functions, respectively. The full spectral function is calculated from $A_{L, L}=i\left(\mathrm{G}_{L, L}-\mathrm{G}_{L, L}^{\dagger}\right)$ and the left spectral function from $A_{L, L}^{L}=G_{L, L} \Gamma_{1,1} G_{L, 1}^{\dagger}$. The Green's function is defined as $\mathrm{G}=(\mathrm{E}-\mathrm{H}-\Sigma)^{-1}$, and the broadening function $\Gamma$ is negative twice the anti Hermitian component of self energy $\Sigma$. The self energies are $\Sigma_{1,1}=-i t_{1,0}$ and $\Sigma_{N, N}=-i t_{N, N+1}$ for the Schottky contact devices [26,27], and $\Sigma_{1,1}=t_{1,0} g_{0,0} t_{0,1}$ and $\Sigma_{1,1}=t_{N, N+1} g_{N+1, N+1} t_{N+1, N}$ for the doped contact devices [28]. Here, $t$ 's are the coupling matrices, and $g_{0,0}$ and $g_{N+1, N+1}$ are the 
surface Green's functions. We use decimation method $[29,30]$ to calculate the surface Green's function

$A_{n}=A_{n-1}\left(E-H_{n-1}\right)^{-1} A_{n-1}$

$B_{n}=B_{n-1}\left(E-H_{n-1}\right)^{-1} B_{n-1}$

$H_{n}^{s}=H_{n-1}^{s}+A_{n-1}\left(E-H_{n-1}\right)^{-1} B_{n-1}$

$H_{n}=H_{n-1}+A_{n-1}\left(E-H_{n-1}\right)^{-1} B_{n-1}+B_{n-1}\left(E-H_{n-1}\right)^{-1} A_{n-1}$.

Here, $n$ is the iteration number. Iterations are repeated until $A_{n}$ and $B_{n}$ are small enough so that the nearest neighbor coupling can be disregarded. Then the surface Green's function can be obtained from $\mathrm{g}^{\mathrm{s}}(E)=\left(E-H_{n}^{\mathrm{s}}\right)^{-1}$. For $g_{0,0}$, the iteration is started with $A_{0}=t_{1,0}$ and $B_{0}=t_{0,1}$. For $g_{N+1, N+1}$, the iteration is started with $A_{0}=t_{0,1}$ and $B_{0}=t_{1,0}$. In both cases, the matrix $H_{0}$ is the unit cell Hamiltonian of the carbon nanotube, and $H_{0}^{s}=H_{0}$. The coherent drain current is calculated from

$I_{D}=\frac{2 e}{h} \int d E T(E)\left(f_{S}-f_{D}\right)$,

Where, $h$ is Planck's constant, and the transmission is calculated from [25]

$$
T(E)=\operatorname{tr}\left[\Gamma_{1,1}\left(A_{1,1}-G_{1,1} \Gamma_{1,1} G_{1,1}^{\dagger}\right)\right] .
$$

The self-consistent loop is started with an initial guess of the potential profile. We generate the initial profile following Ref. [31]. That is, the initial conduction band edge is a step profile with $E_{C}=E_{C S}$ in the source region, $E_{C}=E_{C S}+E_{g} / 2$ under the gate region, and $E_{C}=E_{C S}-e V_{D S}$ in the drain region. Here, $V_{D S}$ is the drain to source bias, $e$ is the electronic charge, and $E_{C S}$ is the conduction band edge relative to the source Fermi level and is calculated from the charge neutrality condition. However, for Schottky contact, the potentials are fixed at the gate, source, and drain terminals, and therefore, our initial potential profile is generated from the Laplace equation. The update profile for the next iteration of self-consistent loop is created using Anderson mixing scheme [32].

\section{NUMERICAL RESULTS AND DISCUSSIONS}

We simulate coaxially gated zero-Schottky-barrier and doped source-drain contacts CNTFETs. The device cross sections used for simulation are shown in Fig. 1. An $(n, 0)$ zigzag CNT is used as the channel material. The devices have a gate length $\mathrm{L}_{\mathrm{g}}$ of $10 \mathrm{~nm}$ and source and drain extensions $\mathrm{L}_{\mathrm{uS}}$ and $\mathrm{L}_{\mathrm{uD}}$ of $30 \mathrm{~nm}$ each. The gate oxide is $\mathrm{SiO}_{2}$ with thickness $\mathrm{t}_{\mathrm{ox}}$ of $2 \mathrm{~nm}$. Poisson solver uses an extended dielectric thickness $\mathrm{t}_{\mathrm{ox}-\mathrm{ex}}$ (or gate metal thickness) of $6 \mathrm{~nm}$. For Schottky contact devices, the source and drain metal extensions $\mathrm{L}_{\mathrm{ex}}$ of $15 \mathrm{~nm}$ are included in Poisson solver to take care of the fringing electric fields. For the doped contact devices, the source and drain contacts are assumed to be uniformly doped. The gate metal is assumed to have the same work function value as the CNT has. For zero-Schottky-barrier contacts, the source and drain metal Fermi functions align with the conduction band of the CNT. Ballistic transport is assumed. We use both uniaxial and torsional strains in our simulation.
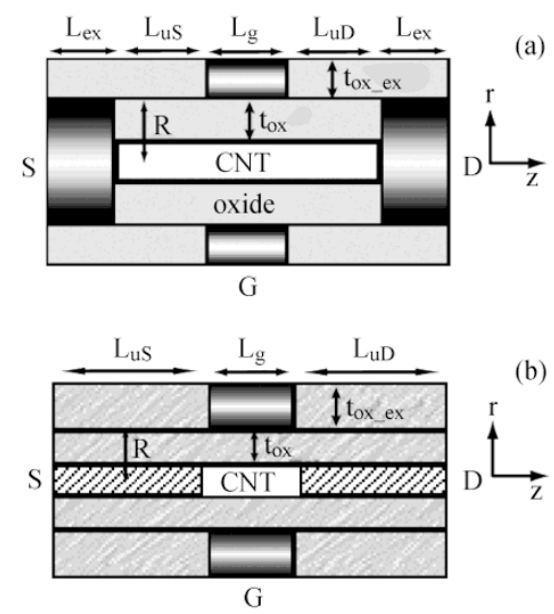

FIG. 1. Device cross sections used for simulation. (a) zero-Schottky-barrier source-drain contacts. (b) doped source-drain contacts.

The band gap variations with uniaxial and torsional strains of $(13,0)$ and $(14,0)$ CNTs that cover two families of CNTs are shown in Fig. 2. The other family, $\bmod (n-m, 3)=0$, is
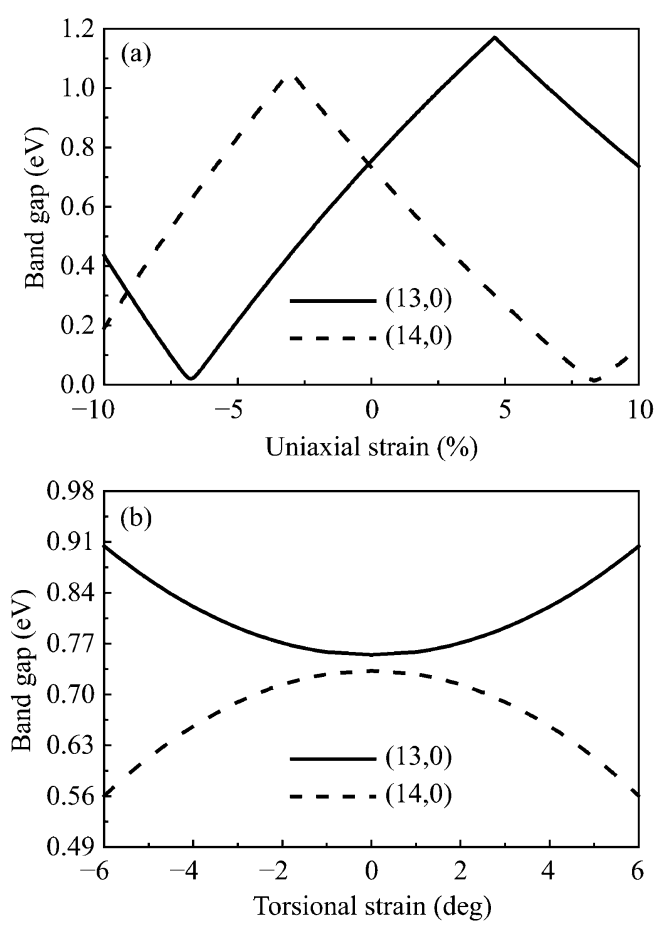

FIG. 2. The band gap variations with (a) uniaxial and (b) torsional strains of $(13,0)$ and $(14,0)$ CNTs. Here, compressive and tensile uniaxial strains are represented as negative and positive strains, respectively. 
not discussed in this study because it has zero band gap at no strain. All the CNTs that have mod $(n-m, 3)=1$ will show the band gap variation similar to $(13,0)$ and similar to $(14,0)$ if mod $(n-m, 0)=2[5,20]$. The band gap of the $(13,0)$ CNT with $2 \%$ uniaxial strain is about $25 \%$ larger than that of the unstrained one. For the $(14,0) \mathrm{CNT}$, this gap is about $26 \%$ smaller than that of the unstrained CNT at $2 \%$ uniaxial strain. A $6^{\circ}$ of torsional strain increases the band gap of the $(13,0)$ CNT by $19 \%$, and decreases the band gap of the $(14,0)$ CNT by $23 \%$. Uniaxial strain on a CNT may either increase or decrease the band gap by applying either tensile or compressive strain. Torsional strain however only increases the band gap of $\bmod (n-m, 3)=1$ CNTs, and decreases the band gap of $\bmod (n-m, 3)=2$ CNTs regardless of whether the torsional angle is positive or negative [20].

From hereon, we will use $(13,0)$ CNT as the channel material to study performance of CNTFETs with strain. The other family shows the opposite behavior of performance metrics with strain compared to the $(13,0)$ devices due to the opposite nature of band gap variation, Fig. 2. The variations of off-state current with strain for $(13,0)$ CNTFETs are shown in Fig. 3. The shape of the off-state current versus strain curves of Schottky-barrier $(13,0)$ CNTFET follows the shape of mod $(n-m, 3)=1$ family reported before [20]. The off-state current in our study is the drain current at zero gate bias with $V_{D S}=0.5 \mathrm{~V}$. The off-state current of both zero-Schotkky-barrier and doped contact devices is almost same at no strain. The off-state current improves with tensile uniaxial strain for $(13,0)$ CNT channel.
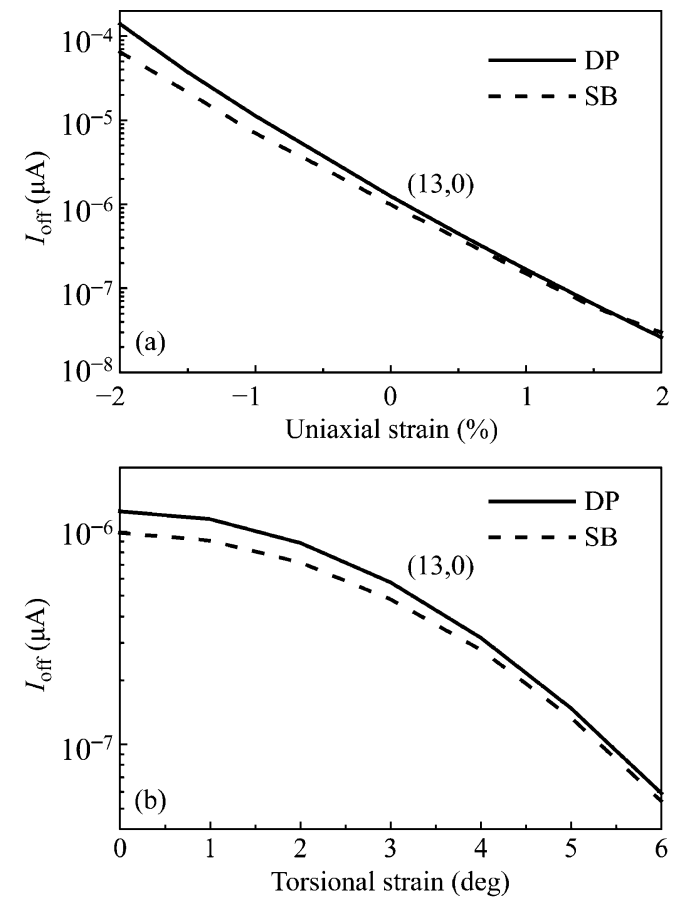

FIG. 3. The off-state current versus strain. The SB means zero-Schottky-barrier and DP means doped source-drain contacts.
The rotational strain improves the off-state current of $(13,0)$ CNT channel. Note that results for positive angles of torsional strain are shown because the band gap variation with torsional strain is symmetric with positive and negative angles. The physics behind the off-state current variation with strain is the modulation of band gap (see Fig. 2) with strain. The off-state current improves with the type of strain that increases the band gap. This is because, with intrinsic channel, the off-state potential barrier height and width increase with larger band gap as shown in Fig. 4. The increased height and width of the barrier reduces the off-state current, mainly the tunneling component of current. The off-state currents are $6.487 \times 10^{-5} \mu \mathrm{A}$, $9.9 \times 10^{-7} \mu \mathrm{A}$, and $3 \times 10^{-8} \mu \mathrm{A}$ for the $(13,0)$ CNT devices with zero-Schottky-barrier contact at a $2 \%$ compressive strain, at no strain, and at a $2 \%$ tensile strain, respectively. These values for the doped contact devices are $1.4 \times 10^{-4} \mu \mathrm{A}, 1.25 \times 10^{-6} \mu \mathrm{A}$, and $2.6 \times 10^{-8} \mu \mathrm{A}$, respectively. So the strain effect on off-state current is not much sensitive to the contact type. This is also true for the torsional strain.
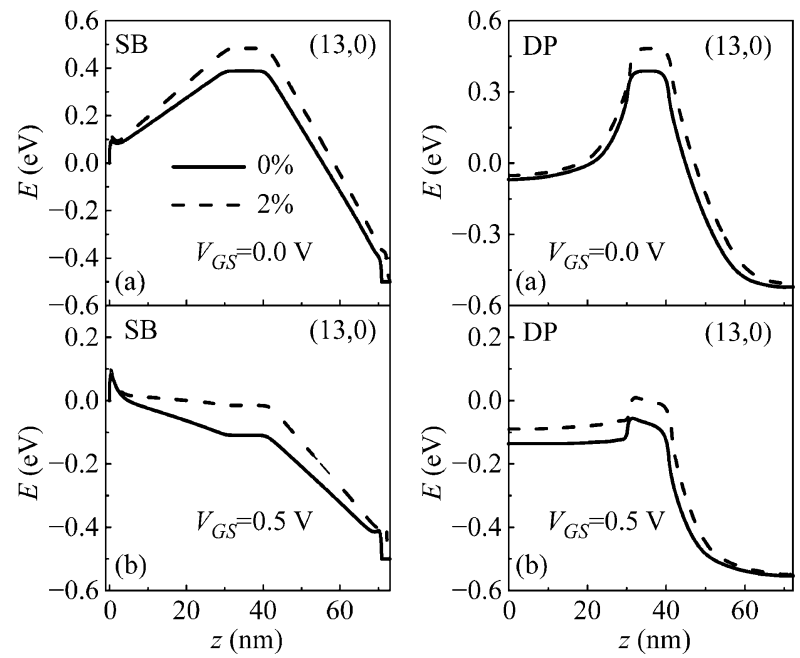

FIG. 4. Conduction band profiles vs channel position in (a) off ( $V_{D S}=0.5 \mathrm{~V}$ and $\left.V_{G S}=0.0 \mathrm{~V}\right)$ state and (b) on $\left(V_{D S}=0.5 \mathrm{~V}\right.$ and $\left.V_{G S}=0.5 \mathrm{~V}\right)$ state. Here, SB means zero-Schottky-barrier, and DP means doped source-drain contacts devices. The channel is a $(13,0)$ CNT. The solid lines are the band profiles of unstrained CNT channel, and the dashed lines are the band profiles of $2 \%$ tensile strained CNT channel

The variations of on-state current with strain are shown in Fig. 5. The on-state current versus strain curves for Schottky-barrier CNTFET have been reported previously [20]. The on-state current in our study is the drain current at $V_{G S}=$ $V_{D S}=0.5 \mathrm{~V}$. The variation trend of on-state current with strain is similar for both types of contact. The on-state current increases with the type of strain that reduces the band gap. This is 
because the on-state potential barrier height reduces with the type of strain that reduces the band gap. This is evident from the band profiles shown in Fig. 4. The on-state current at no strain is much higher in doped contact devices compared to the Schottky contact devices. The on-state currents for Schottky contact devices are $1.78 \mu \mathrm{A}, 0.865 \mu \mathrm{A}$, and $0.47 \mu \mathrm{A}$ at a $2 \%$ compressive strain, at no strain, and at a $2 \%$ tensile strain, respectively. These values for the doped contact devices are $21.6 \mu \mathrm{A}, 9.6 \mu \mathrm{A}$, and $2.23 \mu \mathrm{A}$, respectively. While the improvement of on-state current for the Schottky contact device is from $0.865 \mu \mathrm{A}$ to $1.78 \mu \mathrm{A}$ at a $2 \%$ compressive strain, this is from $9.6 \mu \mathrm{A}$ to $21.6 \mu \mathrm{A}$ for the doped contact device. So the strain effect on on-state current is sensitive to the contact type when current magnitude is compared. However, the percentage change of on-state current with strain is less sensitive to the device contact type. This is also true for the torsional strain.
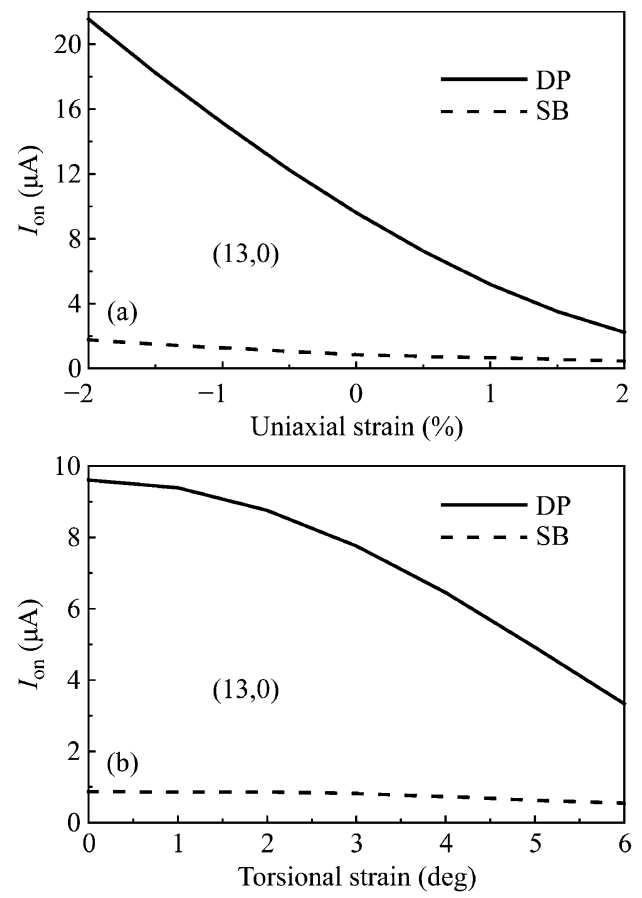

FIG. 5. The on-state current versus strain. Here, SB means zero-Schottky-barrier contact and DP means doped contact.

The on/off current ratio versus uniaxial and torsional strains with Schottky and doped contacts are shown in Fig. 6. The on/off current ratio is better in doped contact devices. The change in on/off current ratio with strain is slightly sensitive to the device contact type, especially in case of torsional strain. This is because the off-state current variation with strain is not sensitive to the contact type, and this sensitivity of on-state current is not significant. For the Schottky contact CNTFETs, the on/off current ratios are $2.74 \times 10^{4}, 8.76 \times 10^{5}$, and $1.85 \times 10^{7}$ at a $2 \%$ compressive strain, at no strain, and at a $2 \%$ tensile strain, respectively. These values for the doped contact devices are
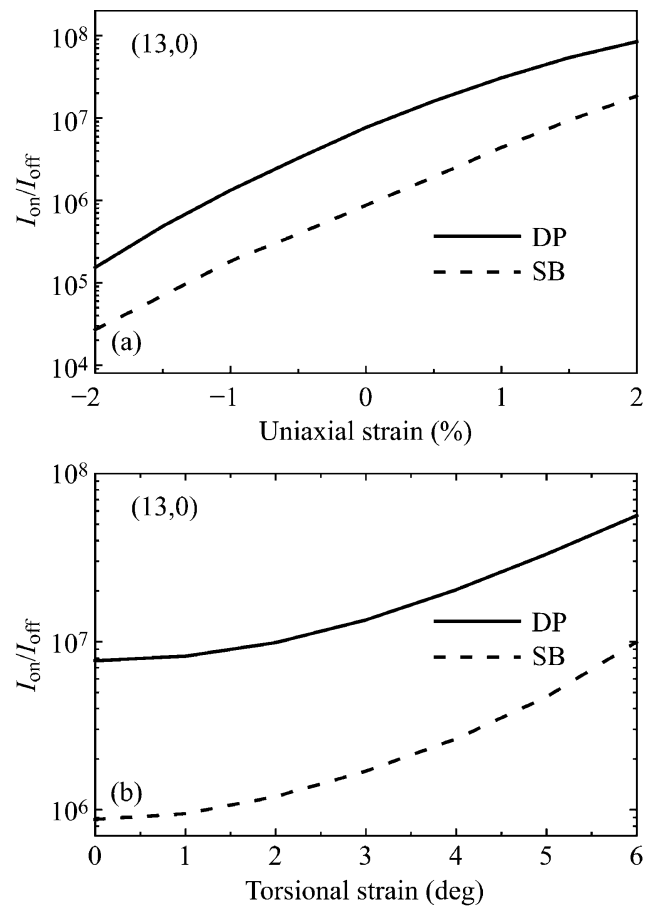

FIG. 6. The on/off current ratio versus strain. Here, SB means zero-Schottkybarrier contact and DP means doped contact.

$1.54 \times 10^{5}, 7.71 \times 10^{6}$, and $8.4 \times 10^{7}$, respectively. The on/off current ratios are $9.96 \times 10^{6}$ and $5.64 \times 10^{7}$ for Schottky contact and doped contact, respectively, with $6^{\circ}$ torsional strain.

The variations of inverse subthreshold slope, defined as $S=\left(\partial \log I_{D} / \partial V_{G S}\right)^{-1}$, with strain are shown in Fig. 7. The inverse subthreshold slope improves with tensile and torsional
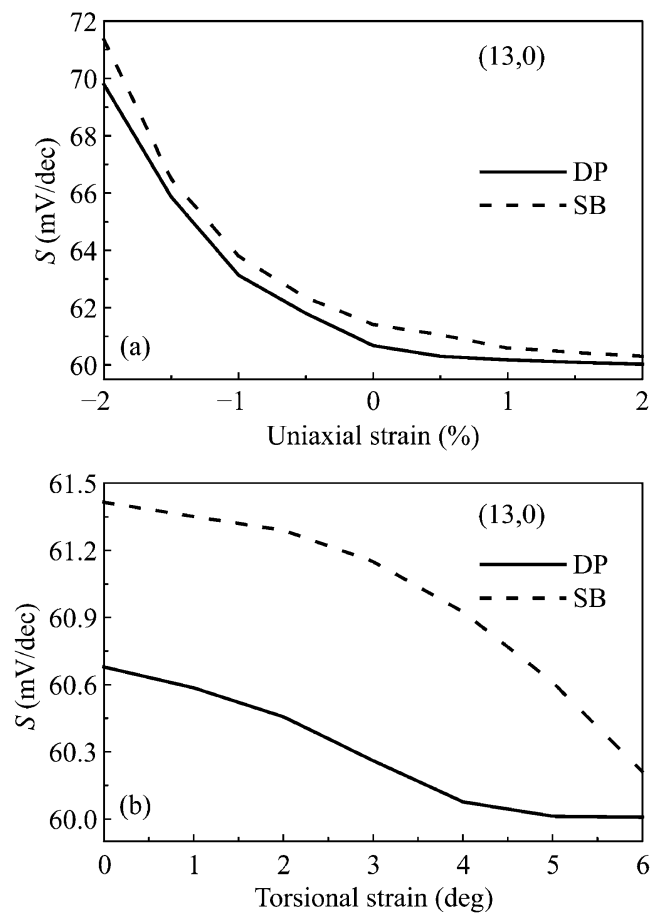

FIG. 7. The inverse subthreshold slope versus strain. Here, SB means zeroSchottky-barrier contact and DP means doped contact.. 
strains. The inverse subthreshold slope is better in doped contact devices, and its change with strain is slightly sensitive to device contact type, especially with torsional strain. The inverse subthreshold slopes are $71.36 \mathrm{mV} / \mathrm{dec}, 61.4 \mathrm{mV} / \mathrm{dec}$, and $60.30 \mathrm{mV} / \mathrm{dec}$ for Schottky contact and $69.78 \mathrm{mV} / \mathrm{dec}$, $60.68 \mathrm{mV} / \mathrm{dec}$, and $60.03 \mathrm{mV} / \mathrm{dec}$ for doped contact at a $2 \%$ compressive strain, at no strain, and at a $2 \%$ tensile strain, respectively. The inverse subthreshold slopes are $60.21 \mathrm{mV} / \mathrm{dec}$ and $60.0 \mathrm{mV} / \mathrm{dec}$ at $6^{\circ}$ torsional strain for Schottky contact and doped contact, respectively.

Finally we compare the switching performance, namely the channel transconductance $g_{m}$, the intrinsic switching delay $\tau_{S}$, and the intrinsic unity current gain frequency $f_{T}$. For this the gate capacitance is calculated from

$$
C_{g}=2 \pi R \int_{0}^{L_{g}} d z \frac{\delta D_{r}}{\delta V_{g}}+2 \pi \int_{t_{o x}}^{t_{o x-e x}} r d r \frac{\delta D_{z}}{\delta V_{g}}
$$

Where, $\mathrm{R}$ is the radius of the dielectric covering $\mathrm{t}_{\mathrm{ox}}$. The first integral takes care of the fluxes emanating from the bottom surface of the gate metal, and the second integral takes care of the fluxes emanating from the two sides of the gate metal facing to the source and drain. This gives the total gate capacitance $C_{g}$ $=C_{g s}+C_{g d}$ which includes the effects of fringing electric fields directly from the gate metal to the source and to the drain. The intrinsic switching delay is calculated from $\tau_{S}=C_{g} V_{D D} / I_{o n}$ and the intrinsic unity current gain frequency from $f_{T}=g_{m} / 2 \pi C_{g}$, where, the transconductance is computed from $g_{m}=\partial I_{D} / \partial V_{G S}$ at $V_{D S}=V_{D D}$.

The on-state transconductance for both types of contact are shown in Fig. 8. The transconductance improves with compressive strain. This dependency is opposite for tensile strain. This change has a clear relationship with the change in band gap with uniaxial and torsional strain. The $g_{m}$ increases with the type of strain that reduces the band gap. The transconductance of doped contact CNTFETs is much higher than that of Schottky contact devices. The values of $g_{m}$ are 5.3 $\mu \mathrm{S}, 4.5 \mu \mathrm{S}$, and $2.65 \mu \mathrm{S}$ with Schottky contact and $116.22 \mu \mathrm{S}$, $88.23 \mu \mathrm{S}$, and $32.08 \mu \mathrm{S}$ with doped contact at a $2 \%$ compressive strain, at no strain, and at a $2 \%$ tensile strain, respectively. The $g_{m}$ reduces with torsional strain. The values of $g_{m}$ are $2.9 \mu \mathrm{S}$ and $43.78 \mu \mathrm{S}$ at a torsional strain of $6^{\circ}$ for Schottky contact and doped contact, respectively. In terms of magnitude change of $g_{m}$, strain has significant sensitivity to the device contact type. However, when the percent change is compared, this sensitivity may not be significant.
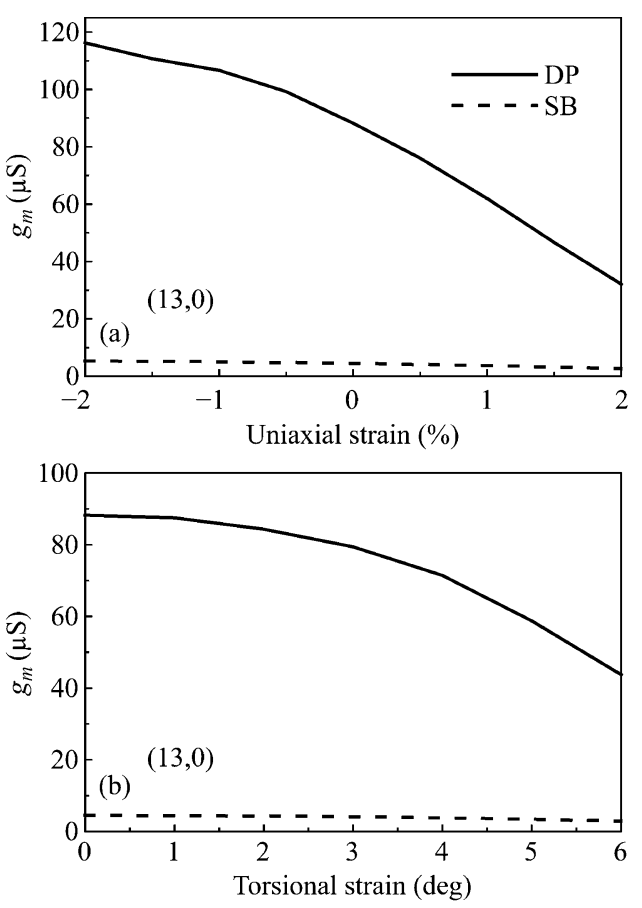

FIG. 8. The on-state transconductance versus strain. Here, SB means zeroSchottky-barrier contact and DP means doped contact.

The switching speed of any device relies on intrinsic switching delay as well as intrinsic cut-off frequency. The on-state intrinsic switching delay and intrinsic cut-off frequency are shown in Fig. 9 and 10, respectively, for the Schottky contact and doped contact. The intrinsic switching delay depends on gate capacitance and on-state current of the device. The switching delay improves with compressive strain.
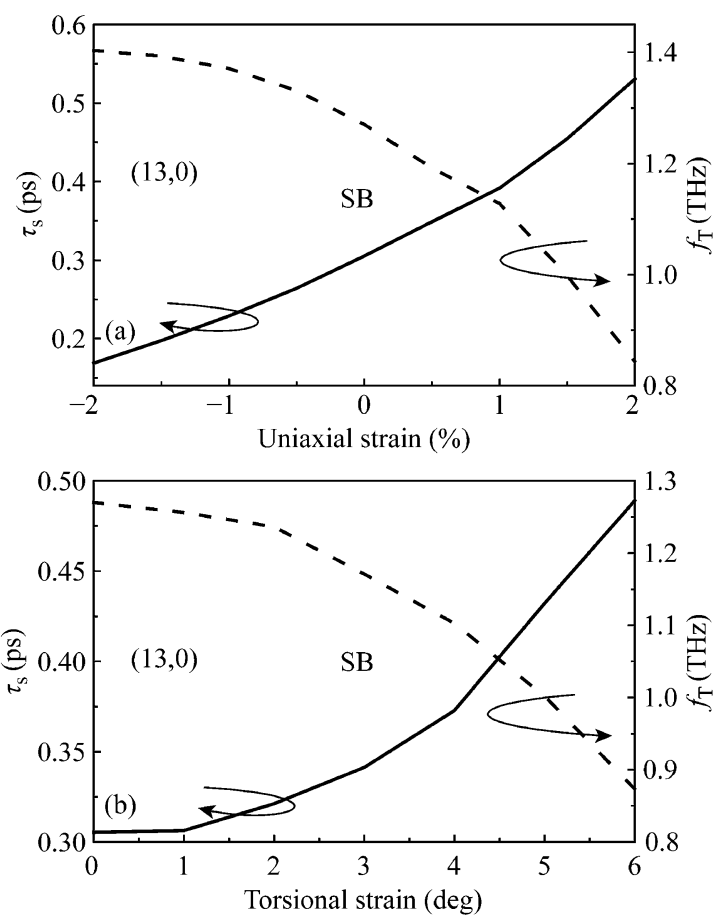

FIG. 9. The on-state intrinsic switching delay and on-state intrinsic cut-off frequency versus strain for zero-Schottky-barrier CNTFETs. 

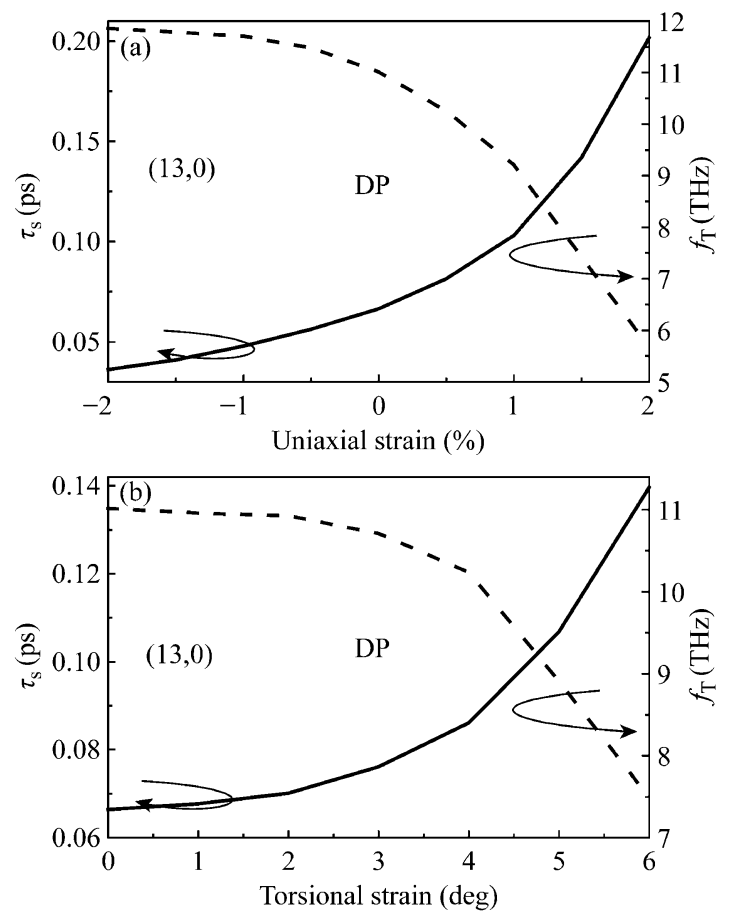

FIG. 10. The on-state intrinsic switching delay and on-state intrinsic cut-off frequency versus strain for doped contact CNTFETs.

Torsional strain degrades the switching delay. The intrinsic switching delays are $0.1689 \mathrm{ps}, 0.3053 \mathrm{ps}$, and $0.5309 \mathrm{ps}$ with zero-Schottky-barrier contact at a $2 \%$ compressive strain, at no strain, and at a $2 \%$ tensile strain, respectively. These values for the doped contact devices are $0.0362 \mathrm{ps}, 0.0664 \mathrm{ps}$, and 0.2017 ps, respectively. The intrinsic switching delays are 0.4892 ps and $0.1396 \mathrm{ps}$ for zero-Schottky-barrier contact and doped contact, respectively, at $6^{\circ}$ torsional strain. Here, also the doped contact CNTFETs outperforms the zero-Schottky-barrier CNTFETs. The switching frequency improves with the type of strain that reduces the band gap. The intrinsic switching frequencies are $1.4035 \mathrm{THz}, 1.2699 \mathrm{THz}$, and $0.8435 \mathrm{THz}$ with zero-Schottky-barrier at a $2 \%$ compressive strain, at no strain, and at a $2 \%$ tensile strain, respectively. For the doped contact devices, they are $11.8574 \mathrm{THz}, 11.0137 \mathrm{THz}$, and $5.6739 \mathrm{THz}$, respectively. The intrinsic switching frequencies are 0.8735 $\mathrm{THz}$ and $7.492 \mathrm{THz}$ for zero-Schottky-barrier and doped contact, respectively, at $6^{\circ}$ torsional strain.

\section{CONCLUSION}

Performance of zero-Schottky-barrier and doped source-drain contacts carbon nanotube transistors is compared using a $\pi$-bond atomistic quantum simulation for different types of strain. The doped source-drain contact transistor has better performance with and without strain applied. The variations of on-state current and switching performance with strain are sensitive to the device contact type. However, strain effect on off-state current, on/off current ratio, and inverse subthreshold slope is almost insensitive to the device contact type.

\section{Received 30 May 2010; accepted 22 June 2010; published online 6 July 2010}

\section{References}

1. T. W. Odom, J. L. Huang, P. Kim and C. M. Lieber, Nature 391, 62 (1998). doi:10.1038/34145.

2. J. W. G. Wildoer, L. C. Venema, A. G. Rinzler, R. E. Smalley and C. Dekker, Nature 391, 59 (1998). doi: $10.1038 / 34139$.

3. C. Kane and E. J. Mele, Phys. Rev. Lett. 78, 1932 (1997). doi:10.1103/PhysRevLett.78.1932.

4. R. Heyd, A. Charlier and E. McRae, Phys. Rev. B 55, 6820 (1997). doi:10.1103/PhysRevB.55.6820.

5. L. Yang, M. P. Anantram, J. han and J. P. Lu, Phys. Rev. B 60, 13874 (1999). doi:10.1103/PhysRevB.60.13874.

6. A. Kleiner and S. Eggert, Phys. Rev. B 63, 073408 (2001). doi:10.1103/PhysRevB.63.073408.

7. L. Yang and J. Han, Phys. Rev. Lett. 85, 154 (2000). doi:10.1103/PhysRevLett.85.154.

8. M. F. Yu, O. Lourie, M. J. Dyer, K. Moloni, T. F. Kelly and R. S. Ruoff, Science 287, 637 (2000). doi:10.1126/ science.287.5453.637.

9. J. P. Salvetat, J. M. Bonard, N. H. Thomson, A. J. Kulik, L. Forro, W. Benoit and L. Zuppiroli, Appl. Phys. A 69, 255260 (1999). doi:10.1007/s003390050999.

10. A. Rochefort, D. R. Salahub and P. Avouris, Chem. Phys. Lett. 297, 45 (1998). doi:10.1016/S0009-2614(98) 01105-1.

11. M. Nardelli and J. Bernholc, Phys. Rev. B 60, 16338 (1999). doi:10.1103/PhysRevB.60.R16338.

12. A. Rochefort, P. Avouris, F. Lesage and D. R. Salahub, Phys. Rev. B 60, 13824 (1999). doi:10.1103/PhysRe vB.60.13824.

13. L. Liu, C. S. Jayanthi, M. Tang, S. Y. Wu, T. W. Tombler, C. Zhou, L. Alexseyev, J. Kong and H. Dai, Phys. Rev. Lett. 84, 4950 (2000). doi:10.1103/PhysRevLett.84.4950.

14. A. Maiti, A. Svizhenko and M. P. Anantram, Phys. Rev. Lett. 88,126805 (2002). $\underline{\text { doi:10.1103/PhysRevLett }}$ $\underline{88.126805 .}$.

15. T. W. Tombler, C. Zhou, L. Alexseyev, J. Kong, H. Dai, L. Liu, C. S. Jayanthi, M. Tang and S. Wu, Nature 405, 769 (2000). doi:10.1038/35015519. 
16. E. D. Minot, Y. Yaish, V. Sazonova, J. Y. Park, M. Brink and P. L. McEuen, Phys. Rev. Lett. 90, 156401 (2003). doi:10.1103/PhysRevLett.90.156401.

17. J. Cao, Q. Wang and H. Dai, Phys. Rev. Lett. 90, 157601 (2003). doi:10.1103/PhysRevLett.90.157601.

18. H. Maune and M. Bockrath, Appl. Phys. Lett. 89, 173131 (2006). doi:10.1063/1.2358821.

19. T. Cohen-karni, L. Segev, O. Srur-Lavi, S. R. Cohen and E. Joselevich, Nature Nanotechnology 1, 36 (2006). doi:10.1038/n nano.2006.57.

20. Y. Yoon and J. Guo, IEEE T. Electron Dev. 54, 1280 (2007). doi:10.1109/TED.2007.896356.

21. J. Knoch, S. Mantl and J. Appenzeller, Solid-State Electron. 49, 73 (2005). doi:10.1016/j.sse.2004.07.002.

22. M. A. Wahab and K. Alam, Jpn. J. Appl. Phys. 49, 025101 (2010). doi:10.1143/JJAP.49.025101.

23. W. A. Harrison, Electronic Structure and the Properties of Solids: The Physics of the Chemical Bond, Freeman, San Francisco, 1990.
24. J. W. Mintmire, D. H. Robertson and C. T. White, J. Phys. Chem. Solids 54, 1835 (1993). doi:10.1016/0022-3697(93) 90296-4.

25. R. Lake, G. Klimeck, R. C. Bowen and D. Jovanovic, J. Appl. Phys. 81, 7845 (1997). doi:10.1063/1.365394.

26. K. Alam and R. K. Lake, J. Appl. Phys. 98, 064307 (2005). doi:10.1063/1.2060962.

27. R. Lake, G. Klimeck, R. C. Bowen, D. Jovanovic, D. Blanks and M. Swaminathan, Phys. Status Solidi B 204, 354 (1997). doi:10.1002/1521-3951(199711)204:1<354:: AID-PSSB354>3.0.CO;2-V.

28. S. Datta, Quantum Transport Atom to Transistor, Cambridge University Press, Cambridge, 2005.

29. M. P. L. Sancho, J. M. L. Sancho and J. Rubio, J. Phys. F 15, 851 (1985).

30. M. Galperin, S. Toledo and A. Nitzan, J. Chem. Phys. 117, 10817 (2002). doi:10.1063/1.1522404.

31. S. Bhowmick and K. Alam, Nano-Micro Lett. 2, 83 (2010).

32. V. Eyert, J. Comput. Phys. 124, 271 (1996). doi:10.1 006/jcph.1996.0059. 